Proceedings of the 2011 Winter Simulation Conference

S. Jain, R.R. Creasey, J. Himmelspach, K.P. White, and M. Fu, eds.

\title{
DEVELOPMENT OF MODEL OF WORKERS' MENTAL PROCESSES RELATED TO ABSENCE NORM AS BEHAVIOR RULE IN AGENT-BASED SIMULATION
}

\author{
Seungjun Ahn \\ SangHyun Lee \\ University of Michigan \\ 2350 Hayward St. \\ Ann Arbor, MI 48109, USA
}

\begin{abstract}
Absenteeism at work adversely affects performance in organizations, and is reportedly complex. Absenteeism is not only a behavior caused by an individual's characteristics, but also a system behavior of organizations, as implied in the notions of absence culture and absence norm. In this paper, it is suggested that the use of agent-based modeling and simulation can be an effective way to study mechanisms of absenteeism. To develop an agent-based simulation, a model of workers' mental processes related to the absence norm - which can be used as behavior rules of agents - is suggested. Finally, results of the simulation with the base model are introduced, and the interplay between the absence norm and absence rate in organizations, as well as the assimilation of workers' perceptions, is discussed.
\end{abstract}

\section{INTRODUCTION}

Absenteeism is defined as the failure to report for work as scheduled (Johns 2008). Absenteeism can adversely affect performance in organizations, yet is a poorly understood organizational phenomenon (Gellatly 1995). Especially labor intensive industries, such as the construction industry, can be vulnerable to absenteeism, as most of the tasks therein are dependent mainly on human resources. For example, it has been reported that potential consequences of absenteeism in construction projects include the interruption of work accomplishments, reduced productivity, increased overtime, and employee fatigue; in sum, a loss of revenue (Hinze, Maxwell, and Hubbard 1985). Therefore, when there is a strong dependency of work processes on a labor force, workers' presence "in the right place at the right time" is crucial to successful work performance.

In this research paper, we examine the use of agent-based modeling (ABM) methodology to study the cause and mechanism of absence behavior of employees. ABM is a relatively new research methodology within organizational science, and has the potential to reveal the mechanisms of social phenomena, and ultimately to offer an insight into how we can lead organizations to a desired state by running computer simulations. Specifically, this paper introduces a model of workers' mental processes related to absence, which can be used as a "behavior rule" in ABM. This model is developed to reflect the formation of absence norm in individuals, and the feedback relation between individuals' behaviors and absence norms in organizations.

The paper begins with a review of the absence research literature, in order to understand how informal mechanism, such as absence norms, can affect individual employees' absence behavior, and how that behavior can in turn affect absence norms. The review is followed by a description of principles and features of ABM, as a modeling approach to study the social phenomena related to absence norms. Then, the development of a model of workers' mental processes as a behavior rule in ABM is introduced. Lastly, simulation results of the ABM are presented and discussed. 


\section{Ahn and Lee}

\section{ABSENCE NORM}

Despite a relatively long history of research on absenteeism compared with many research topics in organizational science (Johns 2008), the main causal factors and the mechanism of absenteeism are still arguable. Although a few studies have been conducted focusing on the organizational factors of absence, the majority of research has focused on personal factors, such as job satisfaction and work attitudes (Gellatly 1995). Unfortunately, meta-analytic reviews of the absence research papers have revealed that, in general, there are only weak correlations between individual-level factors and absenteeism measures (Gellatly 1995; Johns 2008). Understanding these controversial research results, Fitzgibbons (1992) argues that absence researchers should challenge the traditional assumptions about the causes of employee absence, and extend their view to include the roles of both group and organizational factors. Nicholson and Johns (1985) noted that employee absence behavior can be affected by an absence culture, which contains a tolerable level of absence expected by co-workers and management. Also, a few empirical studies have been conducted to see whether perceived absence norms among employees actually affect individual absenteeism or vice versa, and how.

In relatively recent years, there have been increasingly more studies in social science to see absenteeism from this point of view. Since 1990, several scholars in social science have argued that individual absence is under normative influence, meaning absenteeism is not only caused by an individual's situation but is also related to an "absence culture" or "absence norm" within his/her organization. Interest in informal mechanisms like absence culture has steadily gained attention, and now understanding "absence culture" is an important aspect of understanding absenteeism (Nicholson and Johns 1985; Gellatly 1995; Rentsch and Steel 2003). Scholars describe the absence culture and absence norm as follows:

"...absence culture is the set of absence-related beliefs, values, and behavioral patterns that are shared among members of a work group or organizational unit. ... [the absence culture is] shown to account for the different pattern of absences within and between organizational settings (Gellatly and Luchak 1998)", "Employees learn the degree and type of absence behavior that others, usually managers and peers, will accept, and they use this information as a guideline for adjusting their own absence behavior. ... the effect of unit-level absence on individual-level absence was mediated by perceived absence norm (Rentsch and Steel 2003)."

Following these descriptions, absence culture can be seen as a concept that covers every informal aspect of employee absence behavior, including absence-related perception and belief. Likewise absence norm can be seen as a more specific concept that addresses a degree or type of absence behavior accepted as legitimate in an organization. The notions of absence culture and absence norm imply that an individual's absence behavior is not only affected by individual factors, but also by a group property (i.e., culture or norm), the latter being formed through the individual's observation of others' behavior and their aggregated perception of absence norms. This means there is a feedback relation between the individual's behavior and his/her perception. This relation is of particular interest from the perspective of an organization's management, because the existence of a feedback relation implies that a small action within an organization can be amplified, largely impacting the performance of the whole group (Sterman 2000). If we fully understand the mechanism and path of formation of absence culture, then we can gain insight into how to nurture a culture that can help management control absenteeism with minimal cost.

\section{AGENT-BASED MODELING}

From the review of the absence research literature in the above section, we can identify several elements that have to be taken into account in our absence research. First of all, an organization system involves a feedback relation between individuals' absence behavior and their perception of absence norm. Secondly, the system behavior (e.g., the aggregated absence rate or the aggregated absence norm) is a result of workers' interactions among the group (e.g., observation of others' behaviors). Lastly, as the result of individuals' interaction, the whole system has a pattern of absence behavior (e.g., overall high absence rate or periodical fluctuations), that is, from the system's point of view, caused by the interplay between the 


\section{Ahn and Lee}

shared absence norms among members and the absence behavior of the organization. In other words, the aggregate absence behavior of workers, which can significantly affect the work performance, can be seen as a system behavior which emerges from individual workers' interactions, because a worker's absences are socially influenced by other workers' absences.

The notion of complex systems can be applied here. Complex systems are defined as ones that consist of many similar and simple parts, where any one part can be easily understood, while the behavior of the system as a whole defies simple explanation (Flake 1998). Also, complex systems often involve learning and adaptation of one part, which evolves through experiences in interactions with other parts of the same system. In the context of absenteeism, workers can observe other workers' absences and more or less "adapt" their own absence standard to the informal group norm, while also being controlled by the formal standard (i.e., official standard). This dynamic mechanism in absenteeism makes the aggregate absence behavior of the organization complex, thereby making predictions of the absence behavior difficult.

One of the methods used to model this complex system behavior is agent-based modeling (ABM). $\mathrm{ABM}$ has been widely used in various academic domains, such as physical sciences, biology, economics, cognitive science, and anthropology (Epstein and Axtell 1996, Macy and Willer 2002). ABM is a computational model used to both simulate the actions and interactions of autonomous agents, and to observe the holistic behavior of a system (Flake 1998). In other words, ABM is computational modeling which uses a bottom-up approach: system behaviors are not determined, but are emergent, and are based on what the agents in the system perceive to be in their own interest, and their associated actions. In the case of absenteeism research, workers can be modeled as agents who have several properties related to the perception and the action of absence. Also, as a simulation runs, in each time unit, agents can follow a behavior rule that is developed to capture workers' behaviors in the real world. The behavior rule needs to be simple but sufficient in order to capture essential characteristics of workers' mental processes and behaviors.

\section{MODEL OF WORKERS' MENTAL PROCESSES}

As noted earlier, many researchers who are interested in the etiology of absence take into consideration the existence of an absence norm. The absence norm can be a powerful, informal social influence exerted upon individuals in organizations that contributes to consistency within the group (Rentsch and Steel 2003). In other words, absence norm exists as an "implicit agreement" among individuals, who estimate a legitimate level of absence. Here, the basic premise is that workers tend to adapt to the common level of absence in an organization. (Many researchers, mainly from sociology, have discussed the concept that it is human nature to copy the behaviors, beliefs, and strategies of the majority, especially in an information-poor environment. See Heinrich and Heinrich's (2007) Why Humans Cooperate. Note that the reason for human conformity is not discussed in this paper.)

With an extensive literature review and his own research experiences, Friedkin (2001) proposes a mechanism of norm formation and maintenance, which is developed with the basis of theories in social psychology and a formal, mathematical network theory of social influence. He explains his understanding of norms by saying, "norms are usually based on an interpersonal agreement. Hence, most norms are shared norms by virtue of the process that links the development of normative content to the recognition that it is a shared perception," which coincides with our view of the absence norm. Based on the "combinatorial" theories of consensus formation and group decision-making, he proposes a mathematical formula to represent a social influence process in a group of $\mathrm{N}$ persons:

$$
y_{i}^{(t+1)}=a_{i}\left(w_{i l} y_{1}^{(t)}+w_{i 2} y_{2}^{(t)}+\cdots+w_{i N} y_{N}^{(t)}\right)+\left(1-a_{i}\right) y_{i}^{(l)}
$$

Here, $t$ represents time (i.e., step); $N$ represents the number of members; $a$ represents susceptibility of a person $(0<a<1)$; w represents weight of influence of a member; and $y$ represents a perception or belief (e.g., legitimate level of absence, in the case of this research). He uses this formula to formalize the social influence of workers in an organization, and he analyzes the effect of norm by mathematical network theories. 
Though impressive, Friedkin's social influence model is not sufficient for direct application in the ABM for our research purposes. First, it does not reflect on the mental process of recognizing others' norms by observation, nor on the process of decision-making. In other words, Friedkin's model does not detail how workers can see others' attitudes, internalize them, and make accordant behavior decisions. Secondly, it excludes the effect of official standards - he calls them "sanctions" - on workers' behaviors. In order to model a mental process related to absence, we need to consider the effect of official standards, as well as the effect of informal standards such as absence norms, in our model.

In our model, the formation of the absence norm begins with observing other workers' absence behaviors. Workers can observe and learn the degree of absence behavior of others, which we refer to as "reading." If a project is small, every worker is "visible" to every other worker, but otherwise workers observe a fraction of coworkers due to the limit of social relations in larger organizations. It should be noted here that in our model it is assumed that workers can remember others' absence rates over a limited time span. Even if a identical rate of absence of others is observed, the level of "acceptance" of coworkers' absence behaviors can be varied according to the degree of influence those coworkers have, as well as the "susceptibility" of the worker. The "susceptibility" is a personal property representing a worker's degree of tendency to follow the observed coworkers' value. Even when a worker observes a degree of absence of his/her coworkers very different from his/her own, if obstinate and inflexible (i.e., showing low susceptibility), he/she is unlikely to change his/her standard of absence.

In a self-organized group, such as a local community or society, this observed information of others' behaviors can provide a sole guideline of how to behave. However in task-oriented organizations, such as company and project workgroups, group members' behaviors are also controlled by formal standards (i.e., official policies). Each individual can follow the formal standard at a different level, which we refer to as "conformity." The conformity determines the position of an individual's standard between his/her observed absence level and the official allowance level.

Once an individual worker "forms" his/her own absence standard, he/she decides whether or not to be absent on any given day by comparing his/her absence history with his/her absence standard. We assume that absence consideration depends only on the personal characteristic toward absence (i.e., absenceDriver) and that the decision to be absent is made solely by comparing the worker's past absence record with his/her own internalized absence standard. The higher the absence standard than his/her absence history (i.e., when he/she has a large margin of absence according to his/her own standard), the bigger the chance he/she will decide to be absent. By transition from decision to behavior, his/her decision becomes "visible" to others. This entire iterative process, from observation to behavior, happens in a worker's mind every day. It is shown in Figure 1.

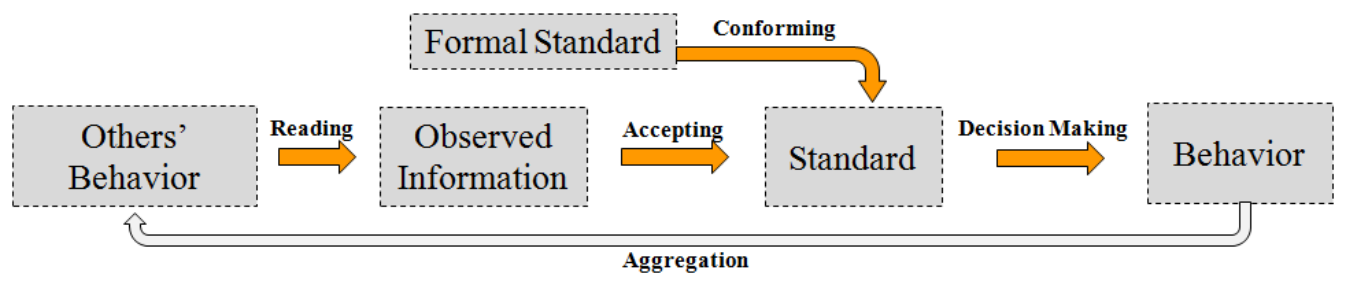

Figure 1: Agent's rule sequence

The behavior rule shown in the Figure 1 can be interpreted as an expansion of the assimilation repertoire of individuals of Friedkin's model. In our model, the process of the agent's behavior includes a complete sequence of observation, formation of norm, and decision making, thereby explaining more comprehensive and disentangled mechanism related to the formation of absence norms. To formalize these processes, a set of equations to define social influences in the formation of the absence norm are proposed as follows: 


$$
\begin{gathered}
\text { Ahn and Lee } \\
X_{i}^{(t)}=w_{i 1} x_{1}^{(t)}+w_{i 2} x_{2}^{(t)}+\cdots+w_{i N} x_{N}^{(t)} \\
y_{i}^{(t+1)}=\left(s_{i}\right) X_{i}^{(t)}+\left(1-s_{i}\right) y_{i}^{(t)} \\
N_{i}^{(t+1)}=\left(c_{i}\right) O^{(t+1)}+\left(1-c_{i}\right) y_{i}^{(t+l)}
\end{gathered}
$$

In support of distinct representation of each step in the agent's behavior process, in addition to Friedkin's model, $x_{i}$ represents the observed monthly absence rate of worker $i$; $\mathrm{X}_{\mathrm{i}}$ represents the aggregated monthly absence rate from observation in worker $i$ 's mind; $s_{i}$ represents susceptibility, which is the tendency to adapt to the level of absence of observed coworkers; $c_{i}$ represents conformity, which is the tendency to adhere to the formal standard; $\mathrm{O}$ is the formal absence standard (unless there is a change in the official absence allowance, $O^{(t)}=O^{(t+1)}=O^{(t+2)}=\cdots=O$ ); and $N_{i}$ represents the individual absence standard of worker $i$. Equations (2) and (3) represent the "reading" and "accepting" processes, respectively, and equation (4) represents the standard forming process with "conforming."

\section{SIMULATION RESULT AND DISCUSSION}

A computational model is created using JAVA in order to implement the conceptual model discussed above. The simulation is comprised of objects which represent agents (i.e., workers) and multiple simulation spaces (for multiple simulation runs). The primary agent is "worker," which is created as an instance of the "Worker" class. Each worker agent has a set of properties, which represent the worker's characteristics related to his/her absence behavior, as listed in Table 1.

Table 1: Properties of Worker Agent

\begin{tabular}{|l|l|l|}
\hline \multicolumn{1}{|c|}{ Name } & \multicolumn{1}{|c|}{ Type and Range } & \multicolumn{1}{c|}{ Description } \\
\hline workerID & int $(\geq 0)$ & Worker ID \\
\hline crewNum & int $(\geq 0)$ & Crew number \\
\hline influenceWeight & double $(0.0-1.0)$ & Influence Weight \\
\hline interactionList & ArrayList & List of acquaintance \\
\hline biasedRead & double $(\geq 1.0)$ & $\begin{array}{l}\text { Level of exaggeration of others' absence when read- } \\
\text { ing }\end{array}$ \\
\hline observedAbsence & double $(\geq 0.0)$ & Aggregated degree of observed monthly absence \\
\hline susceptibility & double $(0.0-1.0)$ & Degree of accepting of group norm \\
\hline perceivedAbsenceNorm & double $(\geq 0.0)$ & Absence norm formed only by observing others \\
\hline conformity & double $(0.0-1.0)$ & $\begin{array}{l}\text { Degree of weighing on formal standard over per- } \\
\text { ceivedAbsenceNorm }\end{array}$ \\
\hline selfStandard & double $(\geq 0.0)$ & $\begin{array}{l}\text { Absence norm resulting from taking into account } \\
\text { both formal standard and informal observed absence } \\
\text { level }\end{array}$ \\
\hline absenceDriver & double $(0.0-1.0)$ & $\begin{array}{l}\text { Degree of individual's inherent or situational causes } \\
\text { of absence }\end{array}$ \\
\hline absenceConsideration & $\begin{array}{l}\text { boolean (true or } \\
\text { false) }\end{array}$ & Whether or not considering absence \\
\hline absenceDecision & $\begin{array}{l}\text { boolean (true or } \\
\text { false) }\end{array}$ & Whether or not deciding absence \\
\hline absenceFor30Days & int $\geq 0)$ & Worker's absence incidents for the last 30 days \\
\hline
\end{tabular}

Once a simulation is initialized, every agent goes through the sequence of behavior, as introduced before. One turn for every agent to go through the sequence represents a discrete time unit, such as one day. When a cause of absence happens to an agent (i.e., when absenceConsideration $=$ true), the agent com- 


\section{Ahn and Lee}

pares his/her own absence history with his/her own absence standard before making a decision (i.e., whether or not absenceFor30Days is larger than selfStandard). When there is a large margin between these two, in other words when one has been absent much less than the absence norm, the agent has a high probability of deciding to be absent that day. When there is little or no margin of absence, the agent has a low probability of absence. An example of the absence probability function depending on the margin can be seen as follows: the probability is $90 \%$ when margin $>5$ days, $80 \%$ when $3<$ margin $\leq 5$, $60 \%$ when $1<$ margin $\leq 3,30 \%$ when $0<$ margin $\leq 1$, and $10 \%$ when margin $\leq 0$. According to the probability determined by this function, the agent's decision whether or not to be absent that day is generated, and this decision is recorded in an array so that other agents can read this behavior the next day.

Running a simulation with the agent-based model - in order to see the relationship between the group absence standard and the aggregate absence rate in the group - allows us to compare changes in these two over the time, as shown in Figure 2. Owing to the initial values set at the beginning of the simulation, there can be a large difference between the average absence standard and the aggregated monthly absence rate of a group. However, as time flows, these two exert an attracting force on each other, as the absence norm has the power to make agents not exceed the norm, and the formation of absence norm depends on every agent's reading of others' absence rates. It is shown that agents settle at a certain level after some time when these two balance out. It should be noted that the "inertia" of these two is dependent on the embedded behavior rule of agents and the initial setting of variables such as susceptibility. The level at which these two ultimately settle depends on many factors, such as initial values for agent variables (i.e., randomness in initial value assignment) and events that happen early on in simulation (i.e., path dependence) (Brown et al 2005).

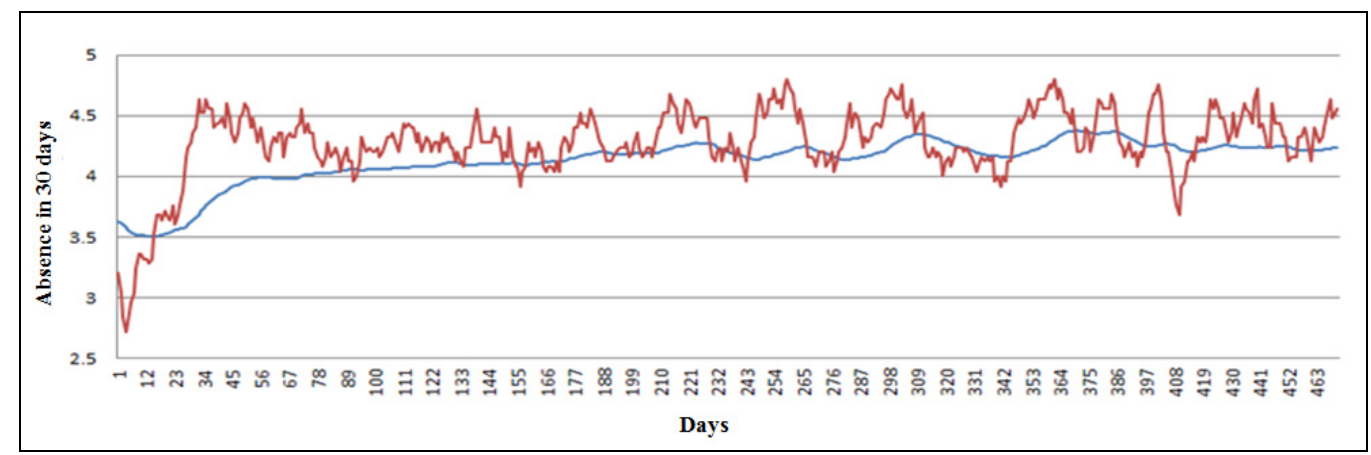

Figure 2: Relationship between Absence Standard and Absence Rate in Group. The red line represents the average monthly absence rate in the group, the blue line represents the average absence standard in the group. These two attract each other, and settle on one level at some point during simulation.

Along with the interrelated change between the absence standard and the absence rate in a group, the distribution of individual agents' absence standard (i.e., selfStandard) also changes as time flows. Figure 3 is created to visualize the change of workers' standards over time. This figure can be seen as a checker board devised to represent a temporal space of all workers, in which each column represents one worker, and each row represents one day (i.e., the size of the checker board is the simulation days by the number of workers. In this simulation case, the checker board is 500 by 100 , because the simulation runs for 500 days and the number of workers is set at 100). To help understand the change of standards over time, the numerical result is color-coded so that bright spots represent a worker at the moment with a low value (i.e., punctual worker), and dark spots represent a worker at the moment with a high value (i.e., slack worker).

At the beginning of the simulation, workers have various levels of absence standard according to the randomly assigned initial value, but as simulation time proceeds, workers go through the sequence of behavior that enforces workers to "assimilate" in terms of the absence standard. We can see this phenomenon as "culturalization of absence" or "formation of group absence norm." In turn, workers" absence rates 
also assimilate, and thus the difference between workers shrinks. Here, again, this assimilation in individuals depends on an individual's characteristics, such as susceptibility and conformity. Also, we can see how the social network takes effect in the formation of absence norm in Figure 3. Since we set the probability of social influence from far-located members to be lower than that of nearby members, workers' standards are affected more by close members than far ones. Therefore, we can see "localized assimilation" from the fact that the results show the averaged absence standard at some areas to be different from other areas after the 500 days of simulation.

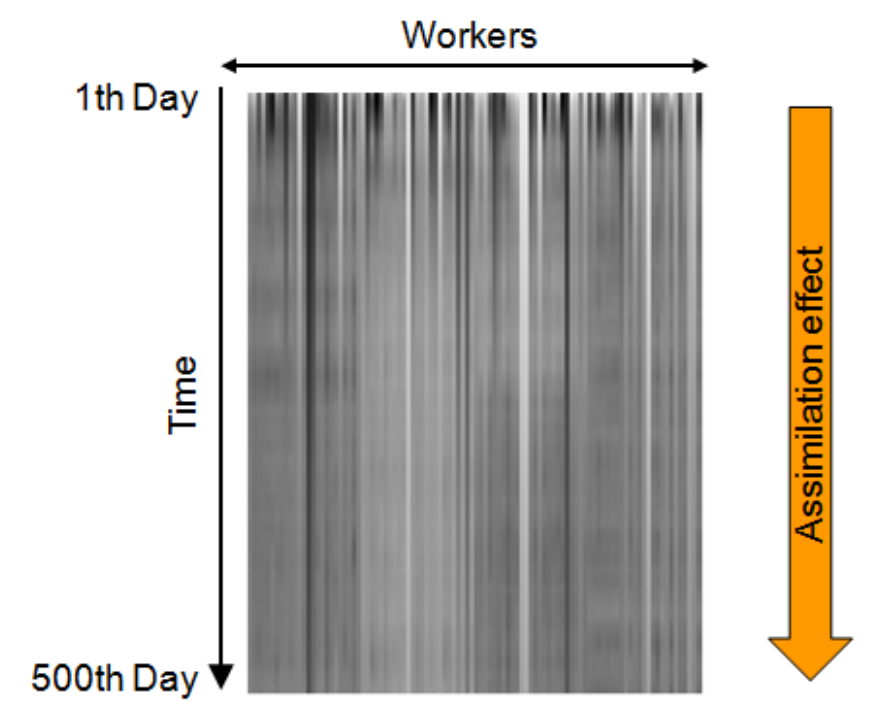

Figure 3: Color-Coded Graph of Individual's Absence Norm. Bright spots represent agents at the moment with a low value of absence standard, and dark spots represent agents at the moment with a high value of absence standard. At the beginning of the simulation, the range of colors of cells is wide, but the range narrows as time goes by due to assimilation.

\section{CONCLUSION}

A high absence rate can adversely affect an organization's work performance. Therefore, controlling absence rate at workplaces is an important issue, as work processes are essentially dependent on the labor force in many industries. The control of workers' absences is an even more important issue in industries that are highly labor intensive, such as the construction industry. This research suggests the use of agentbased modeling (ABM), which enables us to understand the dynamic features of phenomena and investigate an emergence of system behavior from interactions between agents, which ultimately gives us insights into a system's control factors. The objective of this research is to understand the dynamic features of absenteeism using ABM, focusing on the emergence and effect of absence norm.

A model of workers' mental processes has been developed by expanding Friedkin's social influence model. The suggested model consists of three steps: observing others' absence rates, forming an individual absence norm (i.e., self-standard), and making absence decisions. The step of forming of an absence norm can be further divided into two steps: accepting the aggregate absence rate in the group, and positioning one's norm between the group absence rate and the formal standard. A computational model to test this concept is designed, and the model of workers' mental processes is used as the behavior rule of agents in the simulation methodology of ABM. By running a single simulation, we can confirm if the model shows the mechanism of emergence of absence norm and absence culture based on the assimilation among workers' perceptions. 


\section{Ahn and Lee}

With the obtained experience and confidence through this modeling work, there can be many interesting theoretical and empirical research tasks. First of all, we can run multiple simulation experiments in order to see the effect of each model factor on the overall absence behavior of the system. By conducting an extensive set of sensitivity analyses, we can better understand how robust our ABM is, and how reliable the simulation results are. More extensive tests are possible by constructing more systematic, multidimensional parameter spaces to be simulated. Of specific interest - the variable "conformity" determines how self-organized the system is (i.e., how much the overall system absence behavior is affected by the informal absence norm), thereby having a significant impact on the system behavior. Thus, more simultaneous, harmonic changes in pairs of parameters should be tested to better understand factors' effects.

This ABM also brings up numerous topics that can be discussed with experiments with the model. We can conduct various "thought experiments" by varying some affecting factors in the model and observing the results of multiple simulation runs. Examples of these affecting factors include economic status, bias in reading others' behaviors, duration of memory, social network structure, "primacy effect" (which means the first few events are vividly imprinted on one's mind and strongly affect the final consequence), and role of "norm entrepreneurs" (which can be tested by introducing some agents with a unique absence norm in the ABM).

Even though this research proposes a model of workers' mental processes based on theories and empirical research in social psychology and the complex systems study, the model needs to be further reviewed in order to validate constructs in the concept. Specifically, the psychological process in a worker's mind and the decision making process need to be reviewed with extensive studies of previous research in psychology, as well as with a systematic survey of workers' perceptions. If our model can be supported by established theories and compared with actual data related to absenteeism in the industry, we will gain more confidence in it.

\section{REFERENCES}

Brown, D., S. Page, R. Riolo, M. Zellner, and W. Rand. 2005. "Path dependence and the validation of agent-based spatial models of land use." International Journal of Geographical Information Science 19(2):153-174.

Epstein, J. M., and R. Axtell. 1996. Growing Artificial Societies: Social Science from the Bottom Up. Cambridge, MA: MIT Press.

Fitzgibbons, D. E. 1992. "A Critical Reexamination of Employee Absence: The Impact of Relational Contracting, the Negotiated Order, and the Employment Relationship." Research in Personnel and Human Resources Management 10:73-120.

Flake, G. W. 1998. The Computational Beauty of Nature: Computer Explorations of Fractals, Chaos, Complex Systems, and Adaptation. Cambridge, MA: The MIT Press.

Friedkin, N. 2001. "Norm formation in social influence networks." Social Networks 23(3): 167-189.

Gellatly, I. R. 1995. "Individual and group determinants of employee absenteeism: Test of a causal model." Journal of Organizational Behavior 16, no.5:469-485.

Gellatly, I. R., and A. A. Luchak. 1998. "Personal and Organizational Determinants of Perceived Absence Norms." Human Relations 51(8): 1085-1102.

Heinrich, J., and N. Heinrich. 2007. Why Humans Cooperate: A Cultural and Evolutionary Explanation, Evolution and Cognition Series. Oxford: Oxford Univ. Press.

Hinze, J., U. Maxwell, and L. Hubbard. 1985. "Absenteeism in Construction Industry." Journal of Management in Engineering 1(4): 188-200.

Johns, G. 2008. "Absenteeism and Presenteeism: Not at Work or Not Working Well.” In The Sage handbook of organizational behavior 1, edited by C.L. Cooper and J. Barling, 160-177. London: Sage.

Macy, M. W., and R. Willer. 2002. "From Factors to Actors: Computational Sociology and Agent-Based Modeling." Annual Review of Sociology 28(1): 143-166. 
Nicholson, N., and G. Johns. 1985. “The Absence Culture and the Psychological Contract-Who's in Control of Absence?" The Academy of Management Review 10(3): 397-407.

Rentsch, J., and R. P. Steel. 2003. "What does Unit-Level Absence Mean? Issues for Future Unit-Level Absence Research." Human Resource Management Review 13:185-202.

Sterman, J. D. 2000. Business Dynamics: Systems Thinking and Modeling for a Complex World. Boston: McGraw-Hill.

\section{AUTHOR BIOGRAPHIES}

SEUNGJUN AHN is a PhD student in the Construction Engineering and Management Program at the University of Michigan. He is interested in studying dynamics in construction organizations, modeling the emergent behavior of organizations by applying Agent-Based Modeling (ABM), and identifying control factors in construction organizations. Along with pursuing his $\mathrm{PhD}$, he is working toward a Certificate of Graduate Studies in Complex Systems offered by the Center for the Study of Complex Systems at the University of Michigan. His email address is esjayahn@umich.edu.

SANGHYUN LEE is an Assistant Professor at the University of Michigan. Before joining the faculty there, Dr. Lee worked as an Assistant Professor at the University of Alberta, Canada, from 2007 to 2010. He earned a Ph.D. in Construction Management and Information Technology from MIT, and worked at CRA International as a consultant and participated in numerous international construction projects while teaching Project Management at MIT (2006-2007). Dr. Lee's main research interest is the understanding and management of construction dynamics through the design and development of mechanisms, models, and systems that enhance proactive management of mega construction projects. His e-mail is shdpm@umich.edu. 